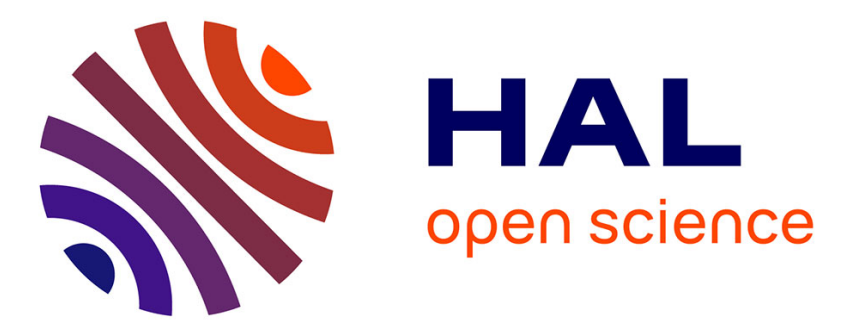

\title{
A semantic annotation framework to assist the knowledge interoperability along a product life cycle
}

Yongxin Liao, Mario Lezoche, Eduardo Rocha Loures, Hervé Panetto, Nacer Boudjlida

\section{- To cite this version:}

Yongxin Liao, Mario Lezoche, Eduardo Rocha Loures, Hervé Panetto, Nacer Boudjlida. A semantic annotation framework to assist the knowledge interoperability along a product life cycle. Advanced Materials Research, 2014, 945-949, pp.424-429. 10.4028/www.scientific.net/AMR.945-949.424 . hal01026591

\section{HAL Id: hal-01026591 https://hal.science/hal-01026591}

Submitted on 24 Jul 2014

HAL is a multi-disciplinary open access archive for the deposit and dissemination of scientific research documents, whether they are published or not. The documents may come from teaching and research institutions in France or abroad, or from public or private research centers.
L'archive ouverte pluridisciplinaire HAL, est destinée au dépôt et à la diffusion de documents scientifiques de niveau recherche, publiés ou non, émanant des établissements d'enseignement et de recherche français ou étrangers, des laboratoires publics ou privés. 


\title{
A Semantic Annotation Framework to Assist the Knowledge Interoperability along a Product Life Cycle
}

\author{
Yongxin Liao ${ }^{1,2,3, a}$, Mario Lezoche ${ }^{2,3, b}$, Eduardo Rocha Loures ${ }^{1, c}$, Hervé \\ Panetto ${ }^{2,3, \mathrm{~d}}$, Nacer Boudjlida ${ }^{4,5, \mathrm{e}}$ \\ ${ }^{1}$ Pontifical Catholic University of Paraná, PPGEPS, Rua Imaculada Conceição, 1155, Curitiba, \\ Paraná, Brazil. \\ 2 Université de Lorraine, CRAN, UMR 7039, Boulevard des Aiguillettes B.P.70239, 54506 \\ Vandoeuvre-lès-Nancy, France; ${ }^{3} \mathrm{CNRS}$, CRAN, UMR 7039, France. \\ ${ }^{4}$ Université de Lorraine, LORIA, UMR 7503, Boulevard des Aiguillettes B.P. 70239, 54506 \\ Vandoeuvre-lès-Nancy, France; ${ }^{5} \mathrm{CNRS}$, INRIA, LORIA, UMR 7503, France. \\ ayongxin.liao@pucpr.br, bmario.lezoche@univ-lorraine.fr, ceduardo.loures@pucpr.br, \\ dherve.panetto@univ-lorraine.fr, enacer.boudjlida @loria.fr
} Keywords: Knowledge Engineering, Semantic Annotation, Semantic Interoperability, Product Life
Cycle, Formalization.

\begin{abstract}
The interoperability among a variety of systems, in or across manufacturing enterprises, has been widely accepted as one of the important factors that affects the efficiency of production. The aim of this study is to deal with the semantic interoperability issues in a product lifecycle management environment. Through the investigation of related works, the need for the formalization of semantic annotation was discovered. This paper addresses this drawback and introduces a framework that uses formalized semantic annotations to assist the knowledge interoperability along a product life cycle.
\end{abstract}

\section{Introduction}

In recent years, more and more manufacturing enterprises have discovered and considered the Product Lifecycle Management (PLM) approach as one of essential solutions to create competitive products. A major current focus in PLM researches is how to ensure a seamless interoperability among different enterprise systems at each stage of a Product Life Cycle (PLC) [1]. Interoperability is an ability of two or more systems or components to exchange information and to use the information that has been exchanged [2]. However, because stakeholders who operate those systems along a PLC have different backgrounds, heterogeneous expertise, unique knowledge, particular needs and specific practices, which over increase the difficulty to achieve interoperability in semantic level [3].

Ontology is a kind of common agreement on the conceptualization of terms in a specific domain of interest [4]. One of the widely recognized methods to deal with semantic gaps is to semantically enrich the exchanged information through an ontology. There exist many studies that highlighted and proved the advantages of applying semantic annotations on various kinds of models, which represent a product from different perspectives. For example, research [5] and [6] focused on annotating data models to support data integration. Research [7] and [8] developed annotation tools to demonstrate the possibility of sharing and reusing the knowledge that is represented in a computer-aided design model. Research [9] and [10] suggested to annotate business process models with semantic contents for assisting designers and analysts in the management of their models. And more generally, research [11] and [12] proposed high level semantic annotation methods for the semantic enrichment of all kinds of enterprise models. Even though lots of efforts were made for addressing the issues related to semantic interoperability, few researchers have paid attention to the formalization perspective of semantic annotations.

The objective of this study is to deal with the interoperability problems, mainly the issue of semantic interoperability, through introducing a formal semantic annotation framework. This 
framework is then being adapted in a new application scenario, to firstly realize the semantic enrichment of models, to secondly assist the knowledge interoperation, and to lastly show its adaptability.

\section{Methodology}

In this paper, we focus on identifying the essential elements of a semantic annotation through its meta-model and presenting a semantic annotation framework to support the mutual understanding of the semantics that's embedded in the exchanged models along the life cycle of a product.

Knowledge Representation. Knowledge is an awareness of things that brings to its owner the capability of grasping the meanings (semantics) from received information [13]. In our work, it is considered as a kind of intangible thing, which has to be made perceptible and afterward to be expressed under certain representations. Knowledge representation is the result of embodying the knowledge from its owner's mind into explicit forms. It gives a possibility for external entities to perform some specific operations and achieve their particular needs. In this study, all relevant models that are generated by stakeholders through different kinds of enterprise systems are all being considered as knowledge representations.

Semantic Interoperability. It signifies an unambiguous understanding of exchanged knowledge representation considering the point of view of both the senders and the receivers [14]. A model "is often presented as a combination of drawings and text" [15]. The mutual understanding of models requires the comprehension of the semantics of both the "combination of drawing" and the "text". Which is why two aspects of semantics are needed to be made explicit by a semantic annotation: (1) Structure semantics, which describes the interrelations between an annotated element and the other elements that it related to; (2) Domain semantics, which describes the context and the meaning of an annotated element in a selected domain.

Meta-model of the Semantic Annotation. Several important concepts that are used throughout this paper need to be cited. In this study, the targets of semantic enrichment are models, which are named as Target Knowledge Representations (TKRs). Ontologies represent real-world semantics that enable a human or a machine to use meaningful terminologies to perform semantic enrichment on TKRs. They are named as Ontology-based Knowledge Representations (OKRs). And more precisely, two types of ontologies are categorized: (1) PLC-related ontologies, which represent the knowledge that related to a product during one or more stages its life cycle; (2) Meta-model ontologies, which represent the knowledge of how models are constructed based on different specifications. A Semantic Annotation is acting as a bridge to describe the semantic relationships between TKRs and OKRs.

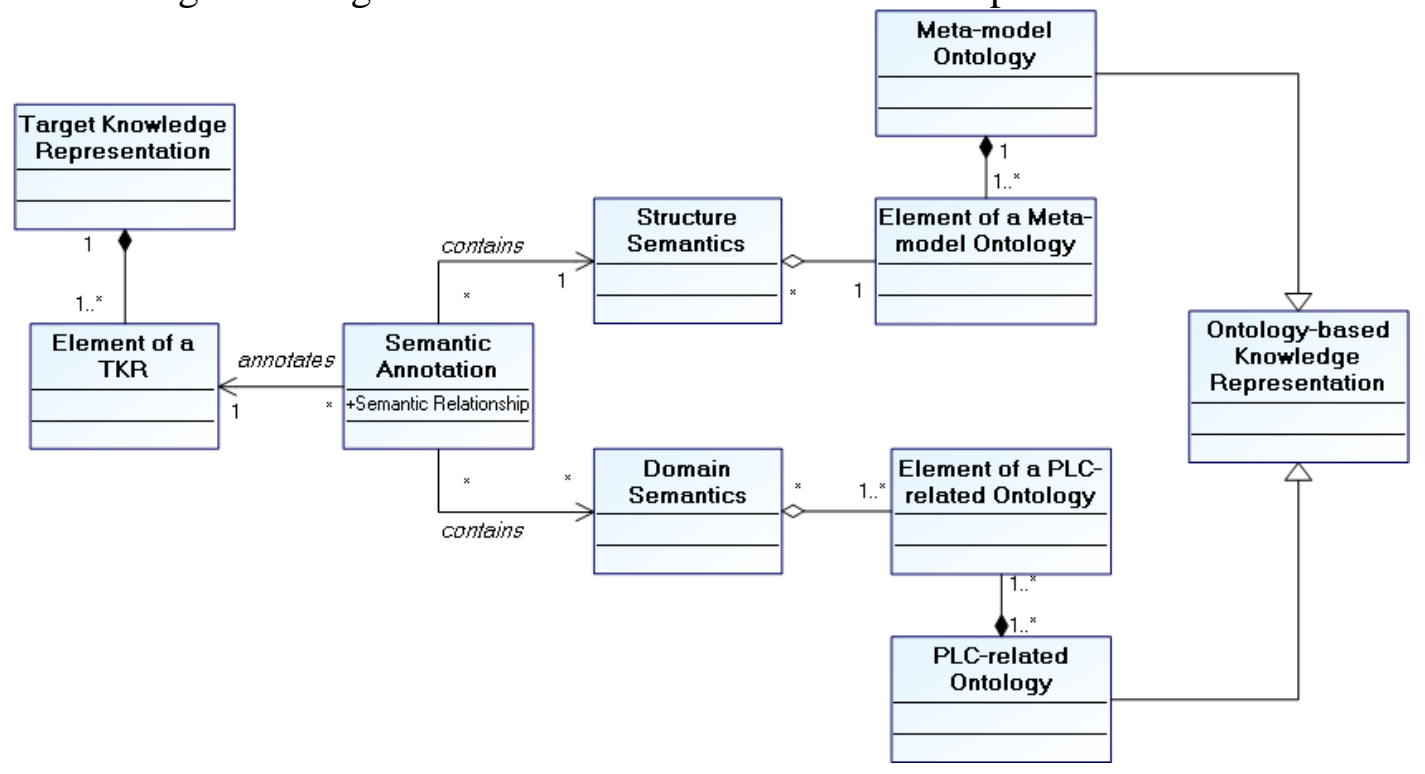

Figure 1 The Meta-model of the Semantic Annotation 
The meta-model of the semantic annotation is given in Fig. 1, which describes its main components (the essential elements) and their relations:

- A "Target Knowledge Representation" is the composition of one or more "Element of a TKR".

- The "Ontology-based Knowledge Representation" is the generalization of the "Meta-model Ontology" and the "PLC-related Ontology".

- A "Meta-model Ontology" is the composition of one or more "Element of a Meta-model Ontology".

- A "PLC-related Ontology" is the composition of one or more "Element of a PLC-related Ontology".

- An "Element of a TKR" can be annotated by zero or more "Semantic Annotation".

- A "Semantic Annotation" contains one "Structure Semantics".

- A "Semantic Annotation" contains zero or more "Domain Semantics".

- A "Structure Semantics" is the aggregation of one "Element of a Meta-model Ontology"

- A "Domain Semantics" is the aggregation of one or more "Element of a PLC-related Ontology".

Semantic Annotation Framework. In the interest of applying formal semantic annotations on multiple TKRs in a PLM environment, as it is shown in Fig. 2, a semantic annotation framework is introduced.

On the left side, there is a series of processes that describes a linear product life cycle. It represents the TKR Creation and Management module, which is composed of a number of enterprise systems. Stakeholders in or across enterprises along the product lifecycle use those systems to create and manage TKRs. Those systems need to provide sufficient APIs to enable the communications between themselves and the Semantic Annotation and Processing Agent module.

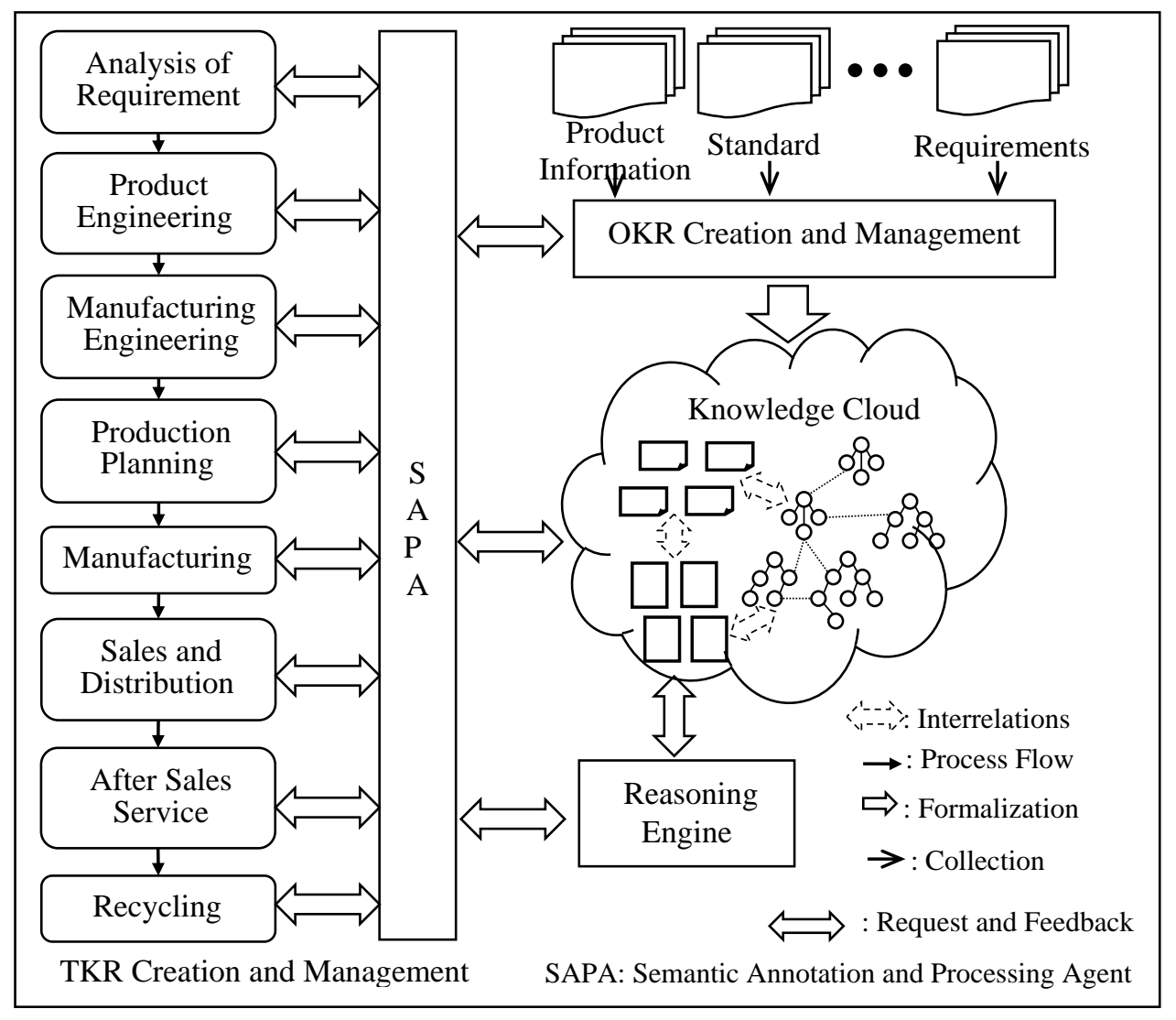

Figure 2 The Proposed Semantic Annotation Framework in a PLM Environment.

On the right side, the $O K R$ Creation and Management module is in charge of capturing, formalizing and managing knowledge related to the life cycle of a product into a knowledge base, 
namely, Knowledge Cloud. The OKRs are supposed to be stored in a platform-independent form, which ensures they can be imported, mapped, merged and interrelated with each other.

The Knowledge Cloud module acts as a knowledge repository. As shown in Fig. 3, three main kinds of knowledge are stored in it:

(1) The OKRs, as it is shown in the Fig. 3 (a), which are structured into a traditional three-levels:

a. Top level ontology. It contains the most common terminology that can be used in different domains.

b. Domain level ontology. It is classified into two aspects: the PLC-related ontologies and the Meta-model ontologies.

c. Application level ontology. Concerning the above-mentioned two aspects of domain level ontologies, the corresponding application level ontologies contain certain specific terms in different enterprises and in different modelling tools respectively.

(2) The semantic annotations, as it can be seen in Fig. 3 (b), which are created by stakeholders along the product lifecycle via using the concepts and relationships in the OKRs

(3) The reasoning rules, as it is shown in the Fig. 3 (c), which are generated based on the OKRs, the implemented semantic annotation schema and corresponding reasoning mechanisms.

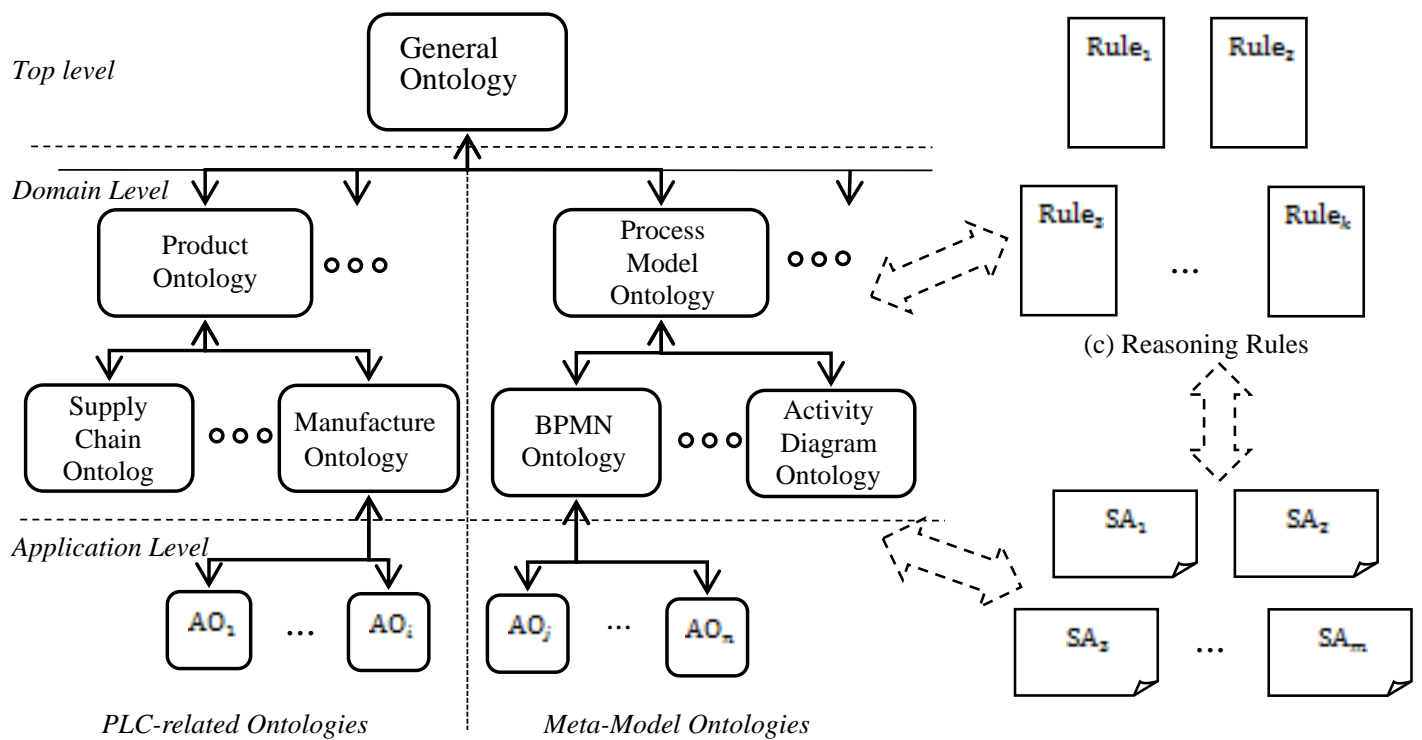

(a) OKRs

(b) Semantic Annotations

Figure 3 The Knowledge Repository: Knowledge Cloud.

The Reasoning Engine module is an external call pattern-matching search engine. It performs inference on the semantic annotations, the OKRs and the reasoning rules according to different stakeholders' requests.

The Semantic Annotation and Processing Agent (SAPA) model is in charge of the definition of semantic relationships. It also acts as a mediator to support the communications (requests and feedbacks) between enterprise systems in different processes of a PLC (TKR Creation and Management module) and the other three modules (Knowledge Cloud module, OKR Creation and Management module and Reasoning Engine module) in the framework.

This framework makes use of formal semantic annotations to describe the semantic relationships between TKRs and OKRs. It makes explicit the implicit semantics of the TKRs, gives possibilities to detect inconsistencies between semantic annotations and to identify possible conflicts among annotated elements, and makes explicit the relations among all disperse TKRs.

\section{Application Scenario}

In order to show the adaptability of this framework, beside the preceding application scenario in our previous research [16], we further prototyped it in a new environment. The lifecycle of a nursery toy 
product, which is manufactured in the Laboratory for Manufacturing Automation ${ }^{1}$ is being chosen As it is shown in Fig. 4, three enterprise systems are selected: The SolidWorks system ${ }^{2}$, which responds of designing the product and generating its technical information; The Bizagi BPM Suite ${ }^{3}$, which in charge of defining and modelling the process models; The Infor Visual Manufacturing system $^{4}$, which is responsible for the production planning and control. They represent the $T K R$ Creation and Management module. For the rest of the framework, the Protégé ontology editor is used as the OKR Creation and Management module, the Microsoft windows folder system is used as the Knowledge Cloud module, the SAP-KM (Semantic Annotation Plugin for Knowledge Management) is considered as the Semantic Annotation and Processing Agency module, and the Jena Reasoner is employed as the Reasoning Engine module.

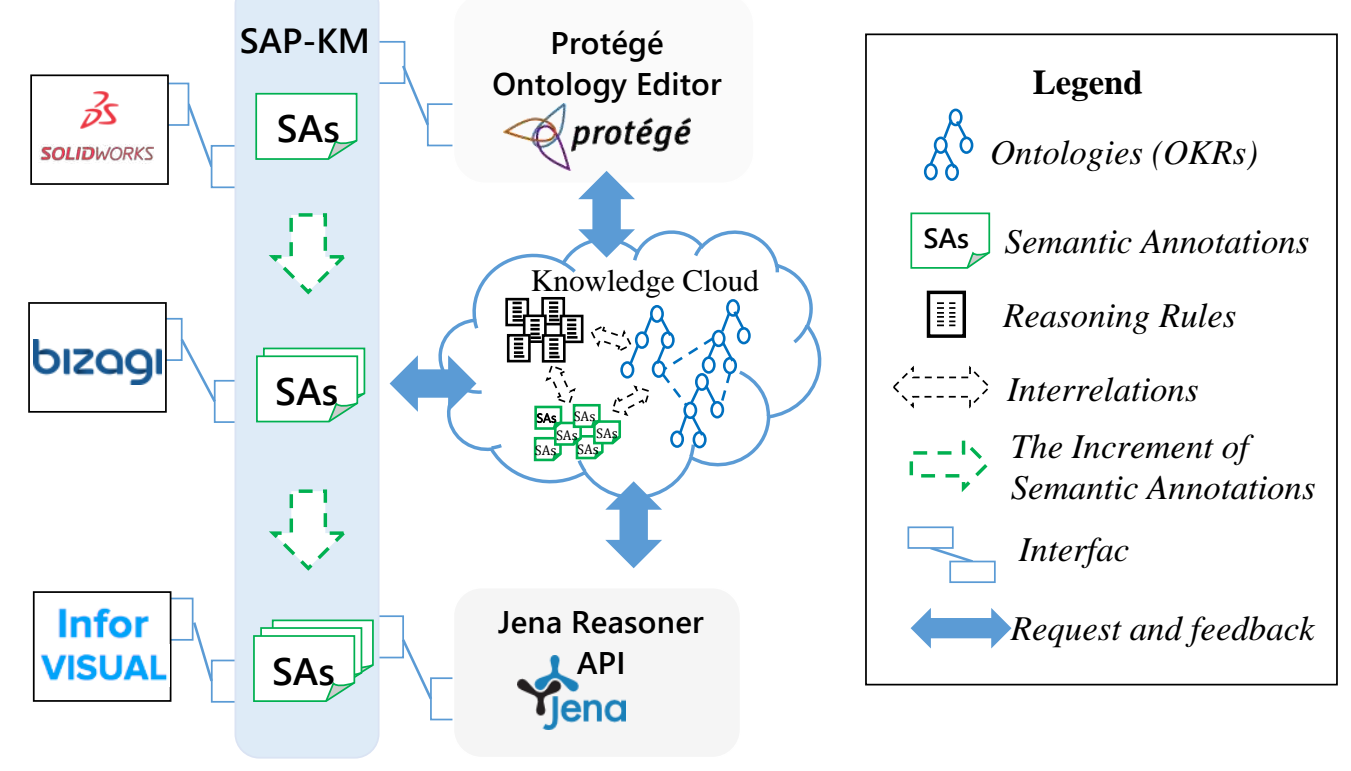

Figure 4 The Application Scenario of the Case Study

Through this prototyped framework, the formalized semantic annotations are contributing in: (1) acquiring the initial semantics that the stakeholders, who manipulate the SolidWorks system, wanted to express; (2) verifying, semi-automatically, the semantic consistency between the contents in the received models and in the developing models in the Bizagi BPM Suite; and (3) guaranteeing the semantics correctness of the under development models for the stakeholders who use the Infor Visual Manufacturing system.

\section{Conclusion and Perspectives}

In summary, we have introduced a semantic annotation meta-model that unambiguously describes its major components and their interrelations, given a semantic annotation framework to apply semantic annotations in a PLM environment, and prototyped this framework in a new application scenario to assist knowledge interoperation. Despite some limitations (such as, the time-consuming ontology creation process, the difficulties in the measurement of semantic relationships, the not perfect annotation process), this paper shows the semantic annotation framework is able to be used in different application scenarios to support the knowledge interoperation along the lifecycle of a product. Furthermore, in future, under the context of the PLM, the potentials of this approach will be used in the following directions: the formalization of requirements from different dimensions; to

\footnotetext{
${ }^{1}$ Laboratory for Manufacturing Automation: http://www.pucpr.br/graduacao/engproducao/curitiba/las01.php

${ }^{2}$ SolidWorks: https://www.solidworks.com/

${ }^{3}$ Bizagi BPM Suite: http://www.bizagi.com/en/products/bizagi-bpm-suite/overview-bpm-suite

${ }^{4}$ Infor Visual Manufacturing: http://go.infor.com/visual/
} 
enable the traceability of requirements along a PLC; and the verification of consistencies among changed and existed requirements.

\section{Acknowledgements}

The authors would like to thank the financial support provided by the Charles Hermite Research Federation (CNRS FR 3198) and the "Région Lorraine" local government in France, and by the Coordination of Improvement of Higher Education Personal (CAPES) in Brasil, under the project of

Science without Borders Program.

\section{References}

[1] F. Ameri and D. Dutta, "Product lifecycle management: closing the knowledge loops," Comput.-Aided Des. Appl., vol. 2, no. 5, pp. 577-590, 2005.

[2] A. Geraci, F. Katki, L. McMonegal, B. Meyer, J. Lane, P. Wilson, J. Radatz, M. Yee, H. Porteous, and F. Springsteel, IEEE standard computer dictionary: Compilation of IEEE standard computer glossaries. IEEE Press, 1991.

[3] A. Etienne, E. Guyot, D. V. Wijk, and L. Roucoules, "Specifications and development of interoperability solution dedicated to multiple expertise collaboration in a design framework," Int. J. Prod. Lifecycle Manag., vol. 5, no. 2, pp. 272-294, 2011.

[4] T. R. Gruber, "A translation approach to portable ontology specifications," Knowl. Acquis., vol. 5, no. 2, pp. 199-220, 1993.

[5] F. Song, G. Zacharewicz, and D. Chen, "An ontology-driven framework towards building enterprise semantic information layer," Adv. Eng. Inform., vol. 27, no. 1, pp. 38-50, 2013.

[6] S. Bergamaschi, D. Beneventano, A. Corni, E. Kazazi, M. Orsini, L. Po, and S. Sorrentino, "The Open Source release of the MOMIS Data Integration System.," in SEBD, 2011, pp. 175-186.

[7] M. Attene, F. Robbiano, M. Spagnuolo, and B. Falcidieno, "Characterization of 3D shape parts for semantic annotation," Comput.-Aided Des., vol. 41, no. 10, pp. 756-763, 2009.

[8] C. Li, "Ontology-Driven Semantic Annotations for Multiple Engineering Viewpoints in Computer Aided Design," University of Bath, 2012.

[9] C. Di Francescomarino, "Semantic annotation of business process models," University of Trento, 2011.

[10] Y. Lin, "Semantic annotation for process models: Facilitating process knowledge management via semantic interoperability," 2008.

[11] N. Boudjlida, H. Panetto, S. Baïna, C. Diamantini, J. Krogstie, Y. Lin, J. Sarraipa, N. Zouggar, A. Hahn, and M. Delgado, "DTG4. 2: Experimental Semantic Enrichment of Enterprise Models for Interoperability and its Practical Impact," 2007.

[12] W3C, "Semantic Annotations for WSDL and XML Schema," 2007. [Online]. Available: http://www.w3.org/TR/2007/REC-sawsdl-20070828/.

[13] R. L. Ackoff, "From data to wisdom," J. Appl. Syst. Anal., vol. 16, pp. 3-9, 2010.

[14] S. Pokraev, D. Quartel, M. W. Steen, and M. Reichert, "Semantic service modeling: Enabling system interoperability," in Enterprise Interoperability, Springer, 2007, pp. 221-230.

[15] J. Miller and J. Mukerji, "MDA Guide Version 1.0. 1," Object Manag. Group, vol. 234, p. 51, 2003.

[16] Y. Liao, M. Lezoche, E. Loures, H. Panetto, and N. Boudjlida, "Semantic Enrichment of Models to Assist Knowledge Management in a PLM environment," in On the Move to Meaningful Internet Systems: OTM 2013 Conferences, 2013, pp. 267-274. 